\title{
AN EFFICIENT METHODOLOGY FOR GENERATING SYNTHETIC POPULATIONS WITH MULTIPLE CONTROL LEVELS
}

\author{
Joshua Auld* \\ Ph.D. Candidate \\ Department of Civil and Materials Engineering \\ University of Illinois at Chicago \\ 842 W. Taylor St. \\ Chicago, IL 60607 \\ Phone: 312-996-0962 \\ Fax: 312-996-2426 \\ Email: auld@uic.edu \\ Abolfazl (Kouros) Mohammadian, Ph.D. \\ Associate Professor \\ Dept. of Civil \& Materials Engineering \\ University of Illinois at Chicago \\ 842 West Taylor Street \\ Chicago, IL 60607-7023 \\ Tel: 312-996-9840 \\ Fax: 312-996-2426 \\ Email: kouros@uic.edu \\ * Corresponding Author
}

Paper Submitted for Publication in Transportation Research Record Final Submission Date: March 15, 2010

$$
\begin{gathered}
\text { Word Count: } 6,406+1,000 \quad(2 \text { table and } 2 \text { Figures }) \\
(\text { Text }) \quad 7,406 \text { words }
\end{gathered}
$$




\begin{abstract}
This paper details a new methodology for controlling attributes on multiple analysis levels within a population synthesis program. The methodology determines how both household- and personlevel characteristics can jointly be used as controls when synthesizing populations, as well as how other multiple level synthetic populations, such as firm/employee, household/vehicle, etc. can be estimated. The use of multi-level controls is implemented through a new technique involving the estimation of household selection probabilities based on the probability of observing each household given the required person-level characteristics in each analysis zone. The new procedure is a quick and efficient method for generating synthetic populations which can accurately replicate desired person-level characteristics
\end{abstract}

\title{
1. INTRODUCTION
}

Population synthesis is recognized as an integral component within activity-based modeling. Starting with the development of the TRANSIMS population synthesizer (1), increased focus has been directed at developing synthetic populations for use in travel demand microsimulation $(2,3$, $4,5)$ and many other agent-based microsimulation applications (6, 7). Population synthesis generally utilizes a sample of households at an aggregate geography combined with marginal data on household characteristics at a disaggregate geography to generate a set of households which satisfy known marginals at the small-area level. Population synthesizers often use a well known statistical technique, Iterative Proportional Fitting or IPF (8), and probabilistic selection in order to generate synthetic populations, although other procedures have recently been developed (9). Either way, a population synthesizer creates copies of sample households and locates them geographically in order to replicate the full population of the study area. For a more in depth discussion of the IPF procedure and basic population synthesis techniques see $(1,10$, 11) among others. The original population synthesis program in which the current work is implemented is discussed at length in (12). This program implemented the basic IPF procedure and probabilistic selection and was developed for use in an activity-based model system (13).

The increasing focus on population synthesis has resulted in recognition of some limitations of the basic synthesis method. This paper aims to improve the methodology behind the basic population synthesis routine in order to account for multiple-levels of analysis units/control variables, which was a limitation to earlier population synthesizers. The paper includes a discussion of the literature on the issue, a description of a newly developed method to address it, validation of the new method and evaluations of its computational performance and finally, a discussion of the value of the new method and directions for future work.

\section{PREVIOUS WORK IN POPULATION SYNTHESIS}

The methodology behind most population synthesizers used in travel demand modeling is generally derived from the synthesizer developed by Beckman et al. (1) for the TRANSIMS project, although some recent work has also addressed the Combinatorial Optimization approach $(7,9)$, or combinations/permutations of both $(14,15)$. During the development of different population synthesizers, many limitations of the basic methodology have been observed. Subsequent research has focused on attempts to correct for these deficiencies and extend the usefulness of synthesis methods $(14,16)$. Several problematic issues relating to population synthesis that have been observed at various times include: zero-cell issues arising from using sample data, biases introduced due to rounding the joint-distributions, biases introduced due to simulation and lack of multiple levels of control $(1,9,15,16)$. Different strategies have been 
proposed to address these issues, for example the zero-cell problem has been addressed by "tweaking" the joint-distribution from the IPF procedure $(1,16)$ and by limiting the number of control variable categories $(12,16)$.

The limitation of population synthesis methods to only one analysis-level has recently begun to receive more attention. Traditionally, population synthesizers only consider control variables for one level as joint-distributions between household and person level control variables can not be constructed. Therefore the IPF procedure and selection procedure as found in Beckman et al (1) can not be implemented directly for both household and person level variables simultaneously (16). Researchers have attempted to overcome this in several ways, including household reconstruction methods (15) or using population characteristics to impute household-level distributions (10). Recent work has focused on methods to address the issue directly in the synthesis procedure, rather than as a reconstruction step. Guo and Bhat (16) account for person-level controls by developing joint-distributions for both individuals and households separately, then synthesizing households while considering whether the person or household level constraints would be violated beyond a given threshold, although only the household distribution is considered when drawing households. Ye et al. (14) developed the only previous attempt to directly and simultaneously control on multiple levels of which the authors are aware. They used an iterative reweighting procedure to heuristically solve for household weights considering both household and person constraints together prior to the household selection procedure. The methodology presented here is a new, efficient procedure for considering joint multi-level controls implemented directly in the selection stage, which builds on the basic IPF and household draw procedure and which, to the best of our knowledge, has not been implemented previously. For details of the basic procedure see Auld et al (12). The new procedure is discussed in the following sections.

\section{MULTI-LEVEL CONTROL METHODOLOGY}

This section discusses the methodology used for multi-level control, implemented within the basic population synthesis program described in Auld et al (12). Multi-level control allows population characteristics to be replicated when creating the synthetic population for more than one analysis-level, with one level such as households serving as the base level of analysis and another sub-level which is contained within the base-level. It should be recognized, however, that there is no requirement that the analysis be used only for synthesizing households/individuals. Any situation where marginal and sample data is available for both a base- and sub-level of analysis (i.e. firms/employees, households/vehicles, buildings/tenants, etc.) can be synthesized using the program. The only limitations are that the membership size of the sub-level within the base level must be used as a control (i.e. household size if using household/individual) and the sample data for the base- and sub-levels must be linked by unique identifiers. The second requirement is due to the fact that the program utilizes a procedure where the base-units are generated and its component sub-units are copied with it rather than synthesizing each sub-unit separately. Since the sub-units are copied with the base unit there must be a link between the base- and sub-unit sample data. For clarity the base- and sub-levels of analysis are referred to hereafter as simply household-level and person-level.

\subsection{Household Selection Probability Considering Person-level Constraints}

One feature most population synthesizers share is the creation of synthetic households through probabilistic selection. This procedure involves setting a probability for selecting a sample 
household into the synthetic population based on the sample weight of the household, the number of total households required, the number of households of the current type already generated, etc. This is the basic procedure followed in the synthesizer by Beckman et al (1) and others. Selection probabilities are assigned for households which are then replicated through simulation. The probabilities increase with the weight of the household and decrease as the required frequency of the current household type is reduced through the simulation process. The required frequency of each household type is taken from the estimated household joint distribution created through the IPF process. Population synthesizers may depart from this basic methodology, as in the procedure developed by Ye et al. (14), where the frequencies determined in the IPF procedure are used in a heuristic iterative solution to set household weights such that person-level constraints are satisfied. Even in this case, however, simulation is still used to create the synthetic households using the reweighted IPF results. The general selection probability as described in Beckman et al (1) is shown in Equation 1.

$$
P_{i, c}=\frac{W_{i}}{\sum_{k=1}^{N_{C}} W_{k}}
$$

where,

$P_{i, c} \quad$ probability of selecting household $i$, of household type $c$

$W_{i} \quad$ = household weight for household $i$

This equation states that the probability of selecting the current household $i$ of a given demographic type $C$ is equal to the weight of the current household divided by the sum of the weights of all other households in the sample of the same type. This selection procedure ensures that households with a higher sample weight are selected more frequently when synthesizing the households. This selection probability does not account for differences between households on the person-level. Therefore, a new selection probability, shown in Equation 2, was developed that explicitly accounts for the person-level distribution when synthesizing the households.

$$
P_{i, c}=\frac{W_{i} \prod_{j=1}^{N_{p e r, i}} \frac{M W A Y_{p e r}^{*}\left(v_{1, j}, v_{2 . j}, \ldots v_{n, j}\right)}{N_{\text {remain }}}}{\sum_{k=1}^{N_{C}}\left(W_{k} \prod_{l=1}^{N_{\text {per }, .}} \frac{M W A Y_{\text {per }}^{*}\left(v_{1, k}, v_{2 . k}, \ldots v_{n, k}\right)}{N_{\text {remain }}}\right.}
$$

where,

$P_{i, c} \quad=$ probability of selecting household $i$, of household type $c$

$W_{i} \quad=$ household weight for household $i$

$N_{\text {per }, i} \quad=$ number of people in household $i$

$M W A Y_{p e r}^{*}\left(v_{1, j}, \ldots v_{n, j}\right)=$ remaining cell frequency in zonal person-level joint distribution

$v_{i, j} \quad=$ index of control variable $i$, for person $j$

$N_{\text {remain }} \quad=$ number of individuals not yet created in zone

$N_{c} \quad=$ remaining households in sub-region sample of type $c$ 
The selection probability defined in Equation 2 has the same form as Equation 1, with the addition of the product terms in the numerator and denominator. These product terms are essentially the probability of observing a household composed of each individual household member given the remaining persons to be synthesized according to the person-level joint distribution, $M W A Y^{*}{ }_{p e r}$. This selection probability is derived from a straightforward application of Bayes Theorem, i.e. the probability of selecting the current household $H$, is the probability of observing household $H$ given the current household type $C$. This is equivalent to the probability of observing each member in the household together divided by the sum of the probability of observing each household member together for all households of the same type, assuming no correlation between the probabilities for individual household members. This assumption is generally incorrect in actuality and would cause problems if we were reconstructing households based on individual probabilities. However, since we are only using the individual probabilities to weight household selection this does not matter - even if unlikely households are weighted the same as likely households on the basis of their individual members, the likely household type is naturally more likely to be observed in the sample data and will therefore be more likely to be generated, as expected. So assumption of independent individual probabilities is corrected by the household weighting term to produce the proper results. This can be reduced even further with the proper choice of household-level control variables. This new selection probability allows the household selection procedure to generate households with individuals that most closely match the required person-level joint distribution. This is best demonstrated with an example, shown in Table 1.

In this example, 25 households of the same type are synthesized from a sample of 4 households with the person-level joint distribution shown in Part a). The basic procedure is shown in Part b), where all four households have the same selection since they have the same weight and the person-distribution is ignored, so that the same number of each household is generated with the resultant synthesized person-level distribution clearly not matching the expected. Part c) then, shows the results when the new selection probability is used. Now the households with more frequent person types in the person-level distribution (HH1, HH4) are generated more than the others. Note that in this simple situation the person-distribution is matched exactly. The example shows that with the new selection probability the person-level marginals and joint distribution are matched when the household and person data are consistent.

\subsection{Updated Household Selection Procedure}

The new household selection probability requires much more calculation than the basic household selection probability definition, due to the sum in the denominator the sum of the products of probabilities for each individual. Under the base methodology, the sum of the weights in the household sample can be calculated once before the synthesis procedure begins and the number can be reused, but the new methodology requires the sum to be recalculated every time the probability is calculated, as the product changes whenever a household/person is synthesized. Therefore a new selection procedure was needed to ensure that populations could be synthesized more efficiently. The new procedure is described in this section.

The procedure behind the new synthesis methodology is as follows for each sub-region (i.e. geographical area at which sample data is available):

1. Generate Sub-region level HH and Person Joint Distributions

a. Create sub-region household-level joint distribution from household sample data

b. Create sub-region person-level joint distribution from person sample data 
c. Use IPF to fit household joint distribution to $\mathrm{HH}$ marginals from marginal data

d. Use IPF to fit person joint distribution to person marginals from marginal data

2. Get next geographic zone within the sub-region

3. Generate zone level $\mathrm{HH}$ and Person Joint Distributions

a. Seed zone $\mathrm{HH}$ joint distribution with sub-region joint distribution

b. Seed zone person joint distribution with sub-region joint distribution

c. Use IPF to fit $\mathrm{HH}$ joint distribution to zone marginal data

d. Use IPF to fit $\mathrm{HH}$ joint distribution to zone marginal data

4. Run household selection procedure

a. Get next household, $\mathrm{H}$, randomly from the sub-region sample

b. Calculate household selection probability $P$ using Equation 1 .

c. Make $N$ attempts to add copy of $\mathrm{H}$ with probability $P$, with $N$ as remaining houses of current type needed in $\mathrm{HH}$ joint distribution

d. Reduce cell in zone $\mathrm{HH}$ joint distribution by number of $\mathrm{H}$ added

e. Remove $\mathrm{H}$ from sub-region sample

f. If households remain in sub-region sample, return to a.)

5. Add all removed households back to sub-region sample

6. If iterations are less than max and households still needed, return to 4.)

7. If zones remaining in sub-region, go to 2.)

8. If Sub-regions remaining, get next sub-region and go to 1.) else finish.

The procedure allows for simultaneous household and person control (as described in the previous section) while enhancing the efficiency of the algorithm. In the traditional household selection procedure $(1,16)$, the list of households in the sub-region sample is searched through many times in order to generate the required number of households. The search procedure generally occurs as follows:

1. Get current household from household list

2. Set selection probability based on Equation 1 multiplied by remaining frequency of household type divided by total remaining households

3. Determine if household is added based on selection probability

4. Return to step 1, if households are still needed in zone

This procedure generally requires much iteration through the sub-region household sample, a process which takes a fairly long time to complete when the new selection probability calculation described in Section 3.1 is used. The new selection procedure simply searches once through the sample household list for each zone. For each household in the sample, the procedure calculates the selection probability then makes a number of attempts to copy the household equal to the remaining frequency in the household joint-distribution for the household type. Each time a copy of the household is successfully added, the probability is updated. After all attempts have been made, the household is removed from consideration, so that it does not figure into the selection probability calculation for later households. This continues until the all households in the list have been searched, at which point, the full population is synthesized. Note that the list is searched in random order to ensure that any biases in the ordering of the sample data are not transferred to the synthetic population.

This process guarantees that the full population is synthesized in one pass through the sample list, greatly reducing the computational run time. However, due to random rounding 
during the synthesis procedure (i.e. if 3.4 household are required, this will be realized as either 3 or 4 households), marginal totals are sometimes violated (12). Therefore a marginal constraint is added to the selection procedure at both the household and person level. This constraint takes the form of an additional rule: if a household is to be added, neither the household or any individual within the household can cause any of the household or person-level marginals to be exceeded by more than a user-defined tolerance. If the marginal constraints will be violated the household is not added. This generally leads to the result that less than the full number of households is generated, usually due to inconsistencies and incompatibilities in the data. Therefore the selection procedure is run for up to a user-defined maximum number of iterations, at which point the marginal constraints are relaxed and the full number of households is generated.

Another problem sometimes arises due to the nature of the selection probability. When calculating the probability for a household, if one of the household members is not needed (i.e. has a remaining frequency of zero in the joint-distribution) the selection probability for that household goes to zero. This is an intentional feature of the procedure and is almost always desirable, but can occasionally cause problems when there are incompatibilities between the household and person-level data. For example, zones such as Block Group 170312704002 in Cook County which has 7 households of household-size four but only 20 total people, will cause the selection procedure to fail. In this example, after the fifth household is generated, there are no people left in the person-level joint distribution, so the household selection probability goes to zero and no households are selected no matter how many iterations are run. Therefore, on the final iteration of the procedure, if there are still households remaining to be generated, the program disregards all person-level controls and generates the remaining households based only on the household weights using the selection procedure seen in Equation 1.

\section{PERSON-LEVEL CONTROL VALIDATION RESULTS}

To assess the validity of the new person-level control methodology, a synthetic population created with the new routine was validated against the same population created without personlevel control. The validation for the person-level control procedure was conducted on 846 block groups in the Chicago-land six-county region where household and person-level marginal control incompatibilities were minimal. Note that many block groups had populations less than the population estimated from the household size control variable, an error which causes less than the full number of households to be generated (since all person-level probabilities are set to zero before all of the households are generated). The selected block groups have a total of 553,387 household containing 1,498,482 individuals, approximately $20 \%$ of the total six-county population. These block groups were selected such that there were no group quarters population and the differences between estimated population totals based on the household size control variables and the population totals in the person-level marginals was less than $2 \%$, in order to separate out error due to the procedure from error caused by data issues. Note that block groups with group quarters are excluded from this analysis only because including a marginal variable relating to group quarter status does not add anything to the person-level validation. When generating synthetic populations for actual modeling purposes it is a straightforward, although cumbersome, procedure to add a group quarters control marginal at the household level which enables block groups with substantial group quarters populations to be generated. In this manner, the validations run below are comparing the differences in procedure rather than differences due to data issues. 
Two separate populations were synthesized, one using only household controls referred to as $P O P-H H$ and one with an additional set of person-level controls referred to as $P O P-P E R$. The household controls used for both populations were:

○ Household size -7 categories

- Household Income - 16 categories

○ Household Number of workers - 5 categories

○ Total Household Joint Distribution size - 560 cells

While the person-level controls used in generating POP-PER were:

$\circ$ Gender -2 categories

- Age -8 categories

- Race -7 categories

- Total Person Joint Distribution size - 112 cells

It should be noted that these variables were selected for demonstration purposes only, as the purpose of this exercise is to confirm that using person-level controls improves the personlevel fit results not to validate the use of this particular set of control variables. Any set of household and person level variables for which adequate sample and marginal data exists can be used as the synthesis program is designed to be as general as possible (12).

Both synthetic populations were able to exactly match the total number of households required, with each generating the actual total of 553,387 households. In addition the total number of individuals generated was almost exact for each synthetic population, as expected even for the non-person control population due to the inclusion of a household size variable as a control. The POP-HH population contains $1,500,308$ people, $0.1 \%$ more than required, while the POP-PER population contains $1,487,815$ people, $0.7 \%$ less than required. The marginal fit comparison, in terms of weighted average absolute percent difference (WAAPD) between the known and synthesized marginal totals over all block groups, for both populations is shown in Figure 1. Note that the Native American/Alaskan and Hawaiian categories in the Race control are not shown as these categories represent less than $0.25 \%$ of the population in the region, although both exhibited similar improvement as the other categories.

The person-level comparison, shown in Figure 1a, demonstrates a substantial improvement in fit between the POP-HH and POP-PER marginal totals on the person level, as expected. Overall there is an improvement in fit of between 52\% and 74\% over each personlevel category, showing that the new routine allows a marked improvement in fitting to person level marginal control totals. As seen in the figure, even under person-level control, the average error associated with certain marginal categories can still be large, although always less than with no person control. This is due mainly to rounding errors and difficulty satisfying the marginal constraints for infrequent categories. The largest errors in the marginal fit are seen for the over-85-years-of-age category and the two-or-more-races category for the age and race marginals respectively, which each represent less than $2 \%$ of the total population. In fact all marginal categories which have a WAAPD of over $15 \%$ contain less than $5 \%$ of the population, meaning that the large errors are mostly the result of small category sizes.

The household-level comparison in Figure $1 \mathrm{~b}$ shows that the improvement in marginal fit using person-level controls comes at a minimal cost to the accuracy of the household-level marginals. All marginal control totals are matched fairly precisely in both the POP-HH and 
POP-PER synthetic populations, with larger errors again seen in the less frequent categories. All household marginal categories had under a 7.0\% WAAPD value.

One point about the procedure should be noted regarding the relaxation of the personlevel constraints used in order to ensure convergence when selecting households. It is clear that allowing the person-level constraints to be violated introduces errors into matching the expected person-level marginals, causing most of the differences seen in Figure 1a. However, analysis shows that in general it is a very small number of generated households and individuals which contribute to these violations, so the impacts are most likely not particularly large. For the POPPER synthetic population, on average over $97 \%$ of households ( $2 \%$ s.d.) and $95 \%$ of individuals (3\% s.d.) were generated before the person-level constraints were relaxed.

The previous analysis only shows how the population matches the marginal characteristics. Therefore each synthetic population was also evaluated on how well the required household- and person-level joint distributions were matched. This is evaluated by estimating the Absolute Percent Difference between the synthesized and expected (from IPF) frequencies for each cell in each block group. This value is then averaged over all block groups to get an Average Absolute Percent Difference (AAPD) value for each cell in each joint-distribution. The AAPD values for each synthetic population are then plotted against the average cell frequency, along with a theoretical estimated AAPD from rounding error calculated as shown in Equation 3 below.

$$
\begin{aligned}
A A P D_{i} & =\frac{\sum_{j=1}^{N_{B G}} A P D_{i, j}}{N_{B G}} \\
A P D_{i, j} & =\left(1-p_{i, j}\right) \frac{x_{i, j}-\left(x_{i, j}-p_{i, j}\right)}{x_{i, j}}+p_{i, j} \frac{\left(x_{i, j}+1-p_{i, j}\right)-x_{i, j}}{x_{i, j}}=\frac{2\left(p_{i, j}\right)\left(1-p_{i, j}\right)}{x_{i, j}}
\end{aligned}
$$

where,

$$
\begin{aligned}
& A P D_{i, j}=\text { expected absolute } \% \text { difference from value in cell } i \text { for block group } j \text { from rounding } \\
& A A P D_{i}=\text { average APD for cell } i \text { over all block groups from rounding } \\
& p_{i, j}=x_{i, j}(\bmod 1) \\
& x_{i, j}=\text { value in cell } i, \text { of person - level joint distribution for blockgroup } j
\end{aligned}
$$

Equation 3 states that the expected absolute percent difference for each cell in the jointdistribution for each block group is the probability of rounding the cell down multiplied by the error caused by this plus the probability of rounding the cell up multiplied by the error caused from rounding up, where the probability is determined by the decimal portion of the actual cell value (i.e. a cell value of 1.2 will be rounding down $80 \%$ of the time and rounded up $20 \%$ of the time, so that $80 \%$ of the time the error is $0.2 / 1.2$ or $16.7 \%$ and $20 \%$ of the time the error is $0.8 / 12$ or $67 \%$, for an average of $26.7 \%$ ). The values for each block group are then averaged to get the AAPD value for each cell. These values are plotted, along with the AAPD values from the POP$\mathrm{HH}$ and POP-PER populations in Figures 2 for both the household- and person-level joint distributions. Note that these values are plotted against average cell frequency, so that a cell with an integer average frequency will still have expected average rounding error. 
Figure 2a shows the results of the comparisons of the AAPD values for each cell in the household distribution matrix, for both the POP-HH and POP-PER synthetic populations. The figure shows that the populations produced through both procedures replicate the householdlevel joint distribution reasonably well, with the AAPD values approaching the theoretically expected value due to random rounding. In fact, the population generated with person-controls actually slightly outperforms the base procedure in satisfying the household distribution with an average AAPD over all cells of $89 \%$ compared to $125 \%$ for the $\mathrm{POP}-\mathrm{HH}$ population. This is possibly due to a more targeted search being performed through the use of the person-level controls and constraints.

The results shown if Figure $2 \mathrm{~b}$ show that, as expected, the fit of the POP-PER synthetic population to the person-level joint distribution is much better than the fit of the POP-HH population, due to the use of the person level controls. The overall AAPD improves from 407\% for the POP-HH to $118 \%$ for the POP-PER population, which is a significant improvement. The cell AAPD values for the POP-PER population are generally much closer to the expected rounding error, while large differences can be seen in the POP-HH AAPD. It should be noted that while the POP-PER AAPD values also generally follow the expected pattern of decreasing error with increasing average cell size, this is not the case with the uncontrolled population, with large errors seen even for several cells with large average sizes, which reinforces the problem with not controlling for person level characteristics. This result is not merely due to the error caused by large variances in the household size between zones as this is accounted for in the calculation of the expected AAPD value.

Overall, the validation analyses presented in Figures 1 and 2 show that the additional use of person-level controls when generating a synthetic population improve the fit of the resulting population to known person-level characteristics when compared to the same synthetic population generated without person-level controls. The increase in fit to the person-level known marginal totals and estimated joint-distribution is very substantial, with little to no sacrifice in the ability to match household level characteristics. In fact, the ability to match the household joint-distribution is somewhat improved through the use of the person-level controls.

A final validation exercise was performed to determine if the new, more-efficient selection procedure outlined in Section 3.2 had any negative impact on the fit of the synthetic populations, when compared to the traditional selection procedure. Note that for this validation analysis the selection procedure refers only to the manner in which the sample households are searched, both procedures tested here still use the new household selection probability calculation which accounts for person-level characteristics. Also, since the test is conducted to determine the validity of the selection procedure rather than the overall synthesis procedure, the marginal constraints were turned off when generating the test synthetic populations. Three different synthetic populations were generated for 46 block groups within PUMAs 3408, 3409, 3518 and 3519 in the Chicago region which had no group quarters population and minimal discrepancies between household-size counts and population levels. The three populations were: person-level control under the new selection procedure (PER-NEW), person-level control under the traditional selection procedure (PER-OLD) and no person control (PER-NONE).

To test for potential biases in the new selection procedure, the Freeman-Tukey test statistic was used to compare the fit of the generated household and person joint-distributions to the expected distributions from the IPF procedure for each procedure. The advantages of this statistic for use in analyzing goodness-of-fit for synthetic population have been described in Voas and Williamson (17) and Ryan et al. (7). The test statistic is calculated as: 


$$
\begin{aligned}
& F T^{2}=4 \sum_{i}^{N_{\text {cells }} N_{\text {zones }}} \sum_{j}\left(\sqrt{\hat{u}_{i j}}-\sqrt{u_{i j}}\right)^{2} \\
& F T^{2} \sim \chi^{2}\left(N_{\text {cells }} \times N_{\text {zones }}-1\right)
\end{aligned}
$$

The statistic is four times the sum of the square of the differences between the square root of actual $\left(u_{i j}\right)$ and estimated $\left(\hat{u}_{i j}\right)$ frequencies over all cells $i$ and zones $j$, and has a chi-square distribution. The test statistic is calculated and compared to a critical value for a given significance level from the $\chi^{2}$ distribution to evaluate the fit of the synthesized population to the person-level joint distribution. The results for all three synthetic populations are shown in Table 2 for both the household and person-level distributions at a significance level of 0.05.

According to Table 2 the null hypothesis for the Freeman-Tukey test, i.e. the synthesized joint distribution and joint distribution resulting from IPF at the person level have the same distribution, is accepted for both populations with person-level controls and rejected for the population without controls, while the household-level distribution is matched for all populations. The results in Table 2 clearly show that using person-level controls improves the fit of the synthesized person-level joint distribution to the estimated distribution, while not controlling for person-level characteristics results in poor fit to the estimated distribution, as expected. More importantly, the good fit to the joint-distribution is obtained for both selection procedures. While the fit obtained by using the new procedure is slightly worse than using the traditional procedure, it is still good and results in a run-time of 0.7 minutes to synthesize the 85,590 individuals in the example above as compared to 18.6 minutes using the other procedure. The run-time for synthesizing the entire population in the Chicago region using the traditional procedure assuming the same rates obtained above would be approximately 30 hours for a single run compared to the 1.4 hours achieved using the new procedure. The long run times using the traditional selection procedure combined with the potential need for running multiple different permutations of a synthetic population and for averaging over multiple runs for the same population motivates the use the more efficient selection procedure, although the traditional selection procedure can still be used to generate a final synthetic population in combination with initial testing and development done using the faster procedure. For this reason, both selection procedures are implemented in the actual synthesis program with the choice left to the user.

\section{COMPUTATIONAL PERFORMANCE}

Beyond validating the accuracy of the new methodology, it is necessary to evaluate its computational performance. To determine the performance characteristics of the new algorithm, the run times for generating the synthetic populations described in the previous section, POP-HH and POP-PER were compared with run times for generating the full Chicago population with and without person-level controls. The same program settings, other than the use of person control, were used in each run. Each synthetic population was generated by running the population synthesis program on an Intel Centrino Duo 2.0 GHz processor.

The non-person controlled population, POP-HH, which contained 1,500,308 synthetic individuals, took 13 minutes to generate. In contrast, the population with person-level controls, POP-PER, with 1,487,815 people, took over 28 minutes. For the full populations, the nonperson controlled full population took about 33 minutes to generate 7,972,057 individuals, while the person-controlled full population took 84 minutes to generate $7,889,221$, out of a total actual 
population of 8,091,720. All of the synthetic populations had a household-level joint distribution size of 560 cells and a person-level joint distribution size of 112 cells.

While it is difficult to compare results across different synthesizers, these run times appear to compare favorably as far as the authors can tell. During the validation of the Atlanta Regional Council population synthesizer, a synthetic population of 1.35 million households controlled only at the household level was run in 17.4 minutes with a household-distribution size of 316 cells (18), about half the time it took to synthesize the 2.9 million households in the Chicago region using only household controls in the new synthesizer.

The only comparable results available for synthesizers which control for person level characteristics were presented in Ye et al (14) for a synthetic population of 2.9 million individuals in Maricopa County, Arizona. This synthetic population was generated using a household-distribution size of 280 cells (over 3 control variables) and a person-joint-distribution size of 140 cells (over the same three control variables used in this study but with two additional age categories). The overall runtime was 16 hours, longer than the 1.4 hours to generate the Chicago population of 7.9 million individuals with approximately the same number of control variables and distribution matrix sizes.

\section{CONCLUSIONS}

This paper has detailed the development of a new methodology for using control variables at multiple analysis levels when synthesizing populations with an existing population synthesizer (12). The new procedure improves the fit of the synthesized person-level characteristics when compared to synthesis procedures that do not account for person-level controls. Validation of the new methodology shows the improved fit to the person-controls comes at no cost to the fit against the household-level controls. Additionally, the introduction of a new household selection procedure has greatly increased efficiency while maintaining good fit to the required personlevel controls without some of the run-time issues that are found using other methods. Note that while the discussion in this paper is mostly limited to the household/person synthesis, this methodology can be applied to any analysis with multiple levels of control. Future work is expected on generating shipping firms/vehicles and business firms/employees, for example, using the same synthesis program. In fact, the applicability of the program is limited only by the availability of data. Overall, the new methodology seems to be an improvement on existing population synthesis techniques for controlling characteristics on multiple levels of analysis.

\section{ACKNOWLEDGMENTS}

The authors would like to acknowledge the financial support provided to this project from the Chicago Metropolitan Agency for Planning (CMAP) and the National Science Foundation (NSF) Integrative Graduate Education and Research Traineeship (IGERT) program in Computational Transportation Science at UIC.

\section{REFERENCES}

(1) Beckman, R.J., K.A. Baggerly and M.D. McKay (1996). Creating synthetic baseline populations. Transportation Research Part A, 30(6), 415-429.

(2) Roorda, M. J., E. J. Miller and K. Habib (2007). Validation of TASHA: A 24-Hour Activity Scheduling Microsimulation Model. Proceedings of the 86th Annual Meeting of the Transportation Research Board (CD), Washington, DC, January. 
(3) Bhat, C.R. J.Y. Guo, S. Srinivasan and A. Sivakumar (2004). Comprehensive Econometric Microsimulator for Daily Activity-Travel Patterns. Transportation Research Record: Journal of the Transportation Research Board, 1894, National Research Council, 57-74.

(4) Yagi, S. and A. Mohammadian. (2008). Modeling Daily Activity-Travel Tour Patterns Incorporating Activity Scheduling Decision Rules, Transportation Research Record: Journal of the Transportation Research Board, No. 2076, TRB, National Research Council, Washington D.C., pp. 123-131.

(5) Frick, M. and K.W. Axhausen (2004). Generating Synthetic Populations using IPF and Monte Carlo Techniques: Some New Results. Paper presented at the 4th Swiss Transport Research Conference, March 25-26, 2004.

(6) Wheaton, W.D., Cajka, J.C., Chasteen, B.M., Wagener, D.K., Cooley, P.C., Ganapathi, L., \& et al. (2009). Synthesized population databases: A US geospatial database for agent-based models. RTI Press Publication No. MR-0010-0905.

(7) Ryan, J., H. Moah and P. Kanaroglou (2009). Population Synthesis: Comparing the Major Techniques Using a Small, Complete Population of Firms. Geographical Analysis 41(2), 181-203.

(8) Deming, W.E. and F.F. Stephan (1940). On a least squares adjustment of a sampled frequency when the expected marginal totals are known. Annals of Mathematical Statistics, 11, 427-444.

(9) Voas, D., and P. Williamson. (2000). An Evaluation of the Combinatorial Optimization Approach to the Creation of Synthetic Microdata. International Journal of Population Geography 6(5), 349-366.

(10) Arentze, T., H. Timmermans and F. Hofman (2007). Creating synthetic household populations - Problems and approach. Transportation Research Record: Journal of the Transportation Research Board, 2014, National Research Council, 85-91.

(11) Hobeika, Antoine (2005). TRANSIMS Fundamentals: Chapter 3 Population Synthesizer, Technical report, U.S. Department of Transportation, Washington, D.C., USA, July 2005, available at http://tmip.fhwa.dot.gov/transims/transims_fundamentals/ch3.pdf , accessed August 1, 2007.

(12) Auld, J.A., A. Mohammadian and K. Wies (2009). Population Synthesis with SubregionLevel Control Variable Aggregation. Journal of Transportation Engineering, 135(9), ASCE, Reston, VA.

(13) Auld, J. A. and A. Mohammadian (2009). Framework for the development of the Agentbased Dynamic Activity Planning and Travel Scheduling (ADAPTS) model.

Transportation Letters: The International Journal of Transportation Research, 1 (3), 243-253.

(14) Ye, X., K.C. Konduri, R.M. Pendyala,, B. Sana, P. Waddell (2009). Methodology to Match Distributions of Both Household and Person Attributes in Generation of Synthetic Populations. Proceedings of the 88th Annual Meeting of the Transportation Research Board (DVD), Washington, D.C., January 11-15, 2009. 
(15) Pritchard, D.R. and E.J. Miller (2009). Advances in Agent Population Synthesis and Application in an Integrated Land Use / Transportation Model. Proceedings of the 88th Annual Meeting of the Transportation Research Board (DVD), Washington, D.C., January 11-15, 2009.

(16) Guo, J.Y. and C.R. Bhat (2007). Population Synthesis for Microsimulating Travel Behavior. Transportation Research Record: Journal of the Transportation Research Board, 2014, National Research Council, 92-101.

(17) Voas, D., and P. Williamson (2001). Evaluating Goodness-of-Fit Measures for Synthetic Microdata. Geographical and Environmental Modeling. 5(2), 177-200.

(18) Bowman, J.L and G. Rousseau (2006). Validation of the Atlanta (ARC) Population Synthesizer (PopSyn). Paper prepared for the TRB Conference on Innovations in Travel Modeling, May 21-23, 2006. Austin, TX. 


\section{LIST OF TABLES AND FIGURES}

TABLE 1 Selection Probability Calculation Example

TABLE 2. Comparison of Synthetic Population Fit for Different Selection Procedures

FIGURE 1 WAAPD Comparison for (a) Person and (b) Household-Level Marginals

FIGURE 2 AAPD Comparison for (a) Household and (b) Person-Level Joint Distribution 
TABLE 1 Selection Probability Calculation Example

a) Starting Data

Microdata sample:

HH1: 1 employed male, 1 employed female, weight $=1$

HH2: 1 unemployed male, 1 employed female, weight $=1$

HH3: 1 unemployed male, 1 unemployed female, weight $=1$

HH4: 1 employed male, 1 unemployed female, weight $=1$

Person-Level Joint Distribution:

HH-Level Joint Distribution

Employed Unemployed Total

\begin{tabular}{l|cc|c} 
Male & 20 & 5 & 25 \\
Female & 10 & 15 & 25 \\
Total & 30 & 20 & 50
\end{tabular}

Total

HHSize $=\mathbf{2} \quad 25 \quad 25$

b) Selection with Household-Level Control Only

Selection Probabilities (Equation 1):

$P(H H 1)=\mathrm{P}(\mathrm{HH} 2)=\mathrm{P}(\mathrm{HH} 3)=\mathrm{P}(\mathrm{HH} 4)=1 /(1+1+1+1)=0.25$

Synthesized Person-Level Distribution:

\begin{tabular}{l|cccl} 
& Employed & Unemployed & Total & HH1_count $=0.25 \times 25=6.25$ \\
\cline { 2 - 3 } Male & 12.5 & 12.5 & 25 & HH2_count $=0.25 \times 25=6.25$ \\
Female & 12.5 & 12.5 & 25 & HH3_count $=0.25 \times 25=6.25$ \\
Total & 25 & 25 & 50 & HH4_count $=0.25 \times 25=6.25$
\end{tabular}

c) Selection with Household- and Person-Level Controls

Selection Probabilities (Equation 2):

$$
\begin{aligned}
& P(H H 1)=\frac{(1)[(20 / 50)(10 / 50)]}{(1)[(20 / 50)(10 / 50)]+(1)[(5 / 50)(10 / 50)]+(1)[(5 / 50)(15 / 50)]+(1)[(20 / 50)(15 / 50)]}=0.32 \\
& P(H H 1)=\frac{(1)[(5 / 50)(10 / 50)]}{(1)[(20 / 50)(10 / 50)]+(1)[(5 / 50)(10 / 50)]+(1)[(5 / 50)(15 / 50)]+(1)[(20 / 50)(15 / 50)]}=0.08 \\
& P(H H 1)=\frac{(1)[(5 / 50)(15 / 50)]}{(1)[(20 / 50)(10 / 50)]+(1)[(5 / 50)(10 / 50)]+(1)[(5 / 50)(15 / 50)]+(1)[(20 / 50)(15 / 50)]}=0.12 \\
& P(H H 1)=\frac{(1)[(20 / 50)(15 / 50)]}{(1)[(20 / 50)(10 / 50)]+(1)[(5 / 50)(10 / 50)]+(1)[(5 / 50)(15 / 50)]+(1)[(20 / 50)(15 / 50)]}=0.48
\end{aligned}
$$

Synthesized Person-Level Distribution:

\begin{tabular}{l|ccrl} 
& Employed & Unemployed & Total & HH1_count $=0.32 \times 25=8$ \\
Male & 20 & 5 & 25 & HH2_count $=0.08 \times 25=2$ \\
Female & 10 & 15 & 25 & HH3_count $=0.12 \times 25=3$ \\
Total & 30 & 20 & 50 & HH4_count $=0.48 \times 25=12$ \\
\hline
\end{tabular}


TABLE 2. Comparison of Synthetic Population Fit for Different Selection Procedures

\begin{tabular}{lcccccccc}
\hline & \multicolumn{3}{c}{ Household-Level Distribution } & & \multicolumn{3}{c}{ Person-Level Distribution } \\
\cline { 2 - 3 } Population & Crit Val. & FT $^{\mathbf{2}}(\boldsymbol{\sigma})$ & $\mathbf{H}_{\mathbf{o}}^{\mathbf{4}}$ & & Crit Val. & FT $^{\mathbf{2}}(\boldsymbol{\sigma})$ & $\mathbf{H}_{\mathbf{o}}^{\mathbf{4}}$ \\
PER-NONE & 26,134 & $4,799(54)$ & Accept & & 5,319 & $24,786(434)$ & Reject \\
PER-OLD & 26,134 & $5,734(68)$ & Accept & & 5,319 & & $4,044(106)$ & Accept \\
PER-NEW & 26,134 & $6,651(82)$ & Accept & & 5,319 & & $4,840(102)$ & Accept
\end{tabular}

1. 25,759 degrees-of-freedom for household-level distribution.

2. 5,151 degrees-of-freedom for person-level distribution.

3. $\mathrm{FT}^{2}$ values averaged over 20 runs, standard deviation of $\mathrm{FT}^{2}$ value shown in parentheses.

4. Null hypothesis accepted if $\mathrm{FT}^{2}$ is less than critical value at significance level of 0.05 , i.e. probability of observing $\mathrm{FT}^{2}$ statistic due to random chance is greater than $5 \%$. 


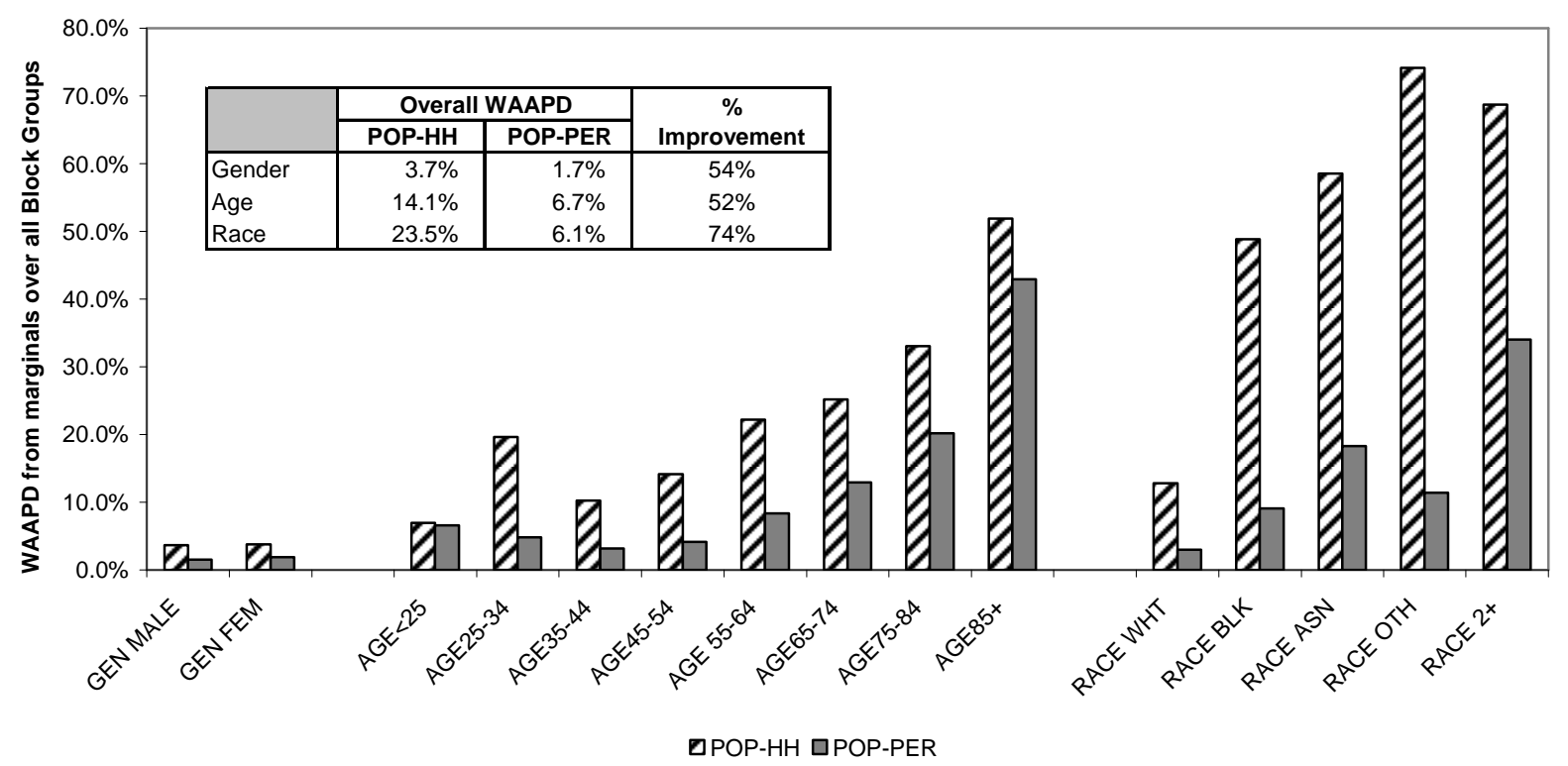

(a)

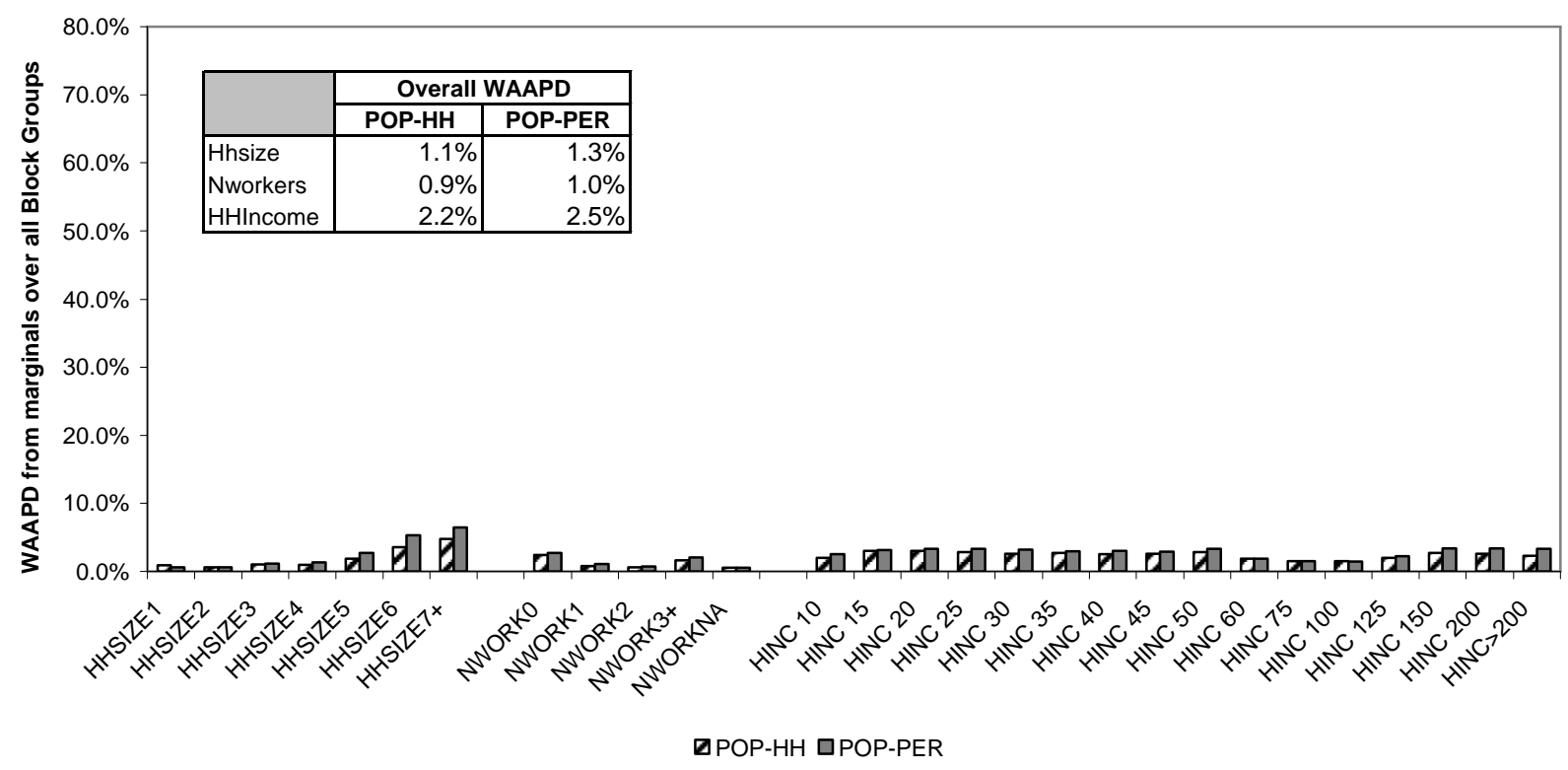

(b)

FIGURE 1 WAAPD Comparison for (a) Person and (b) Household-Level Marginals 


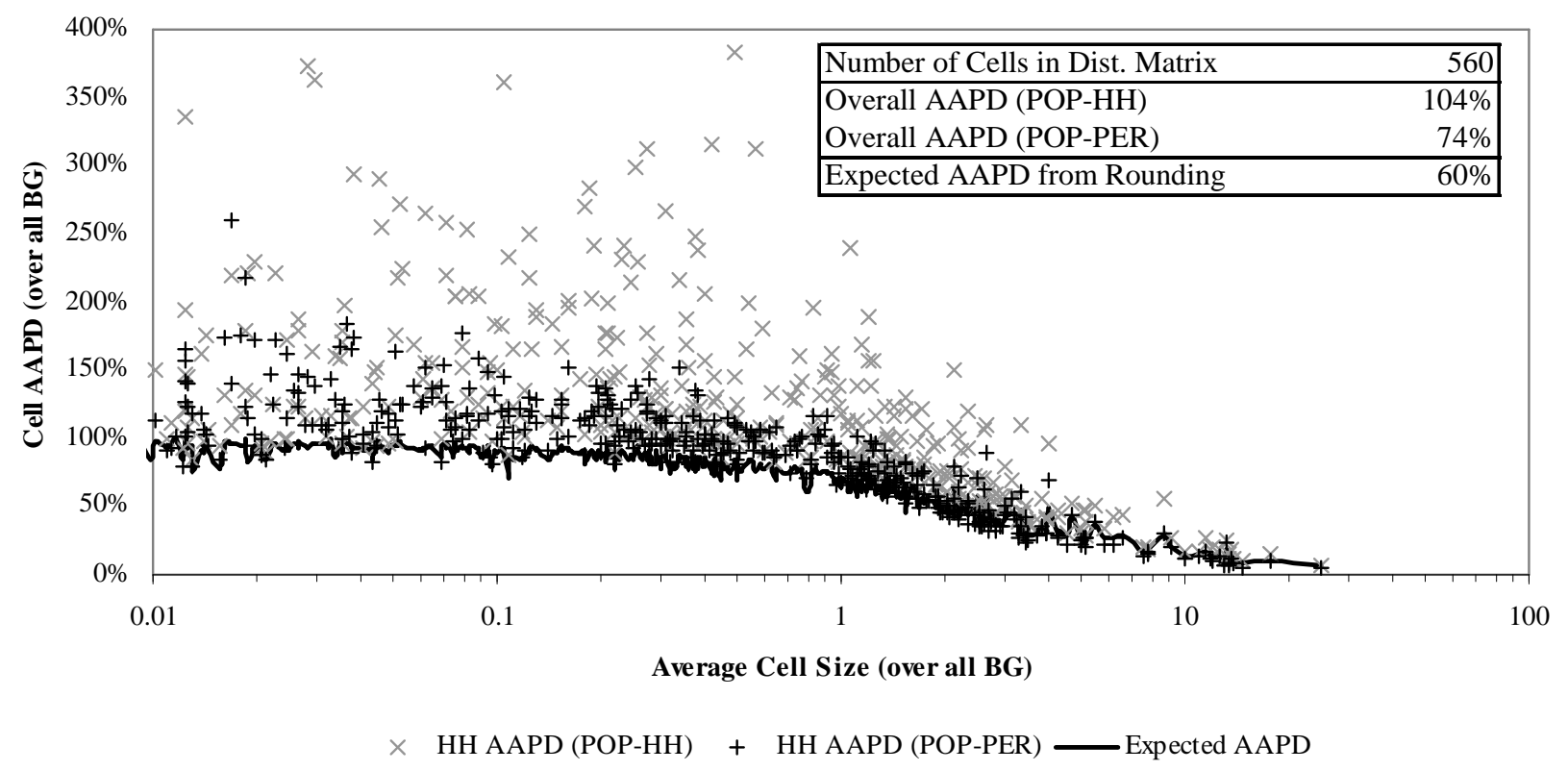

(a)

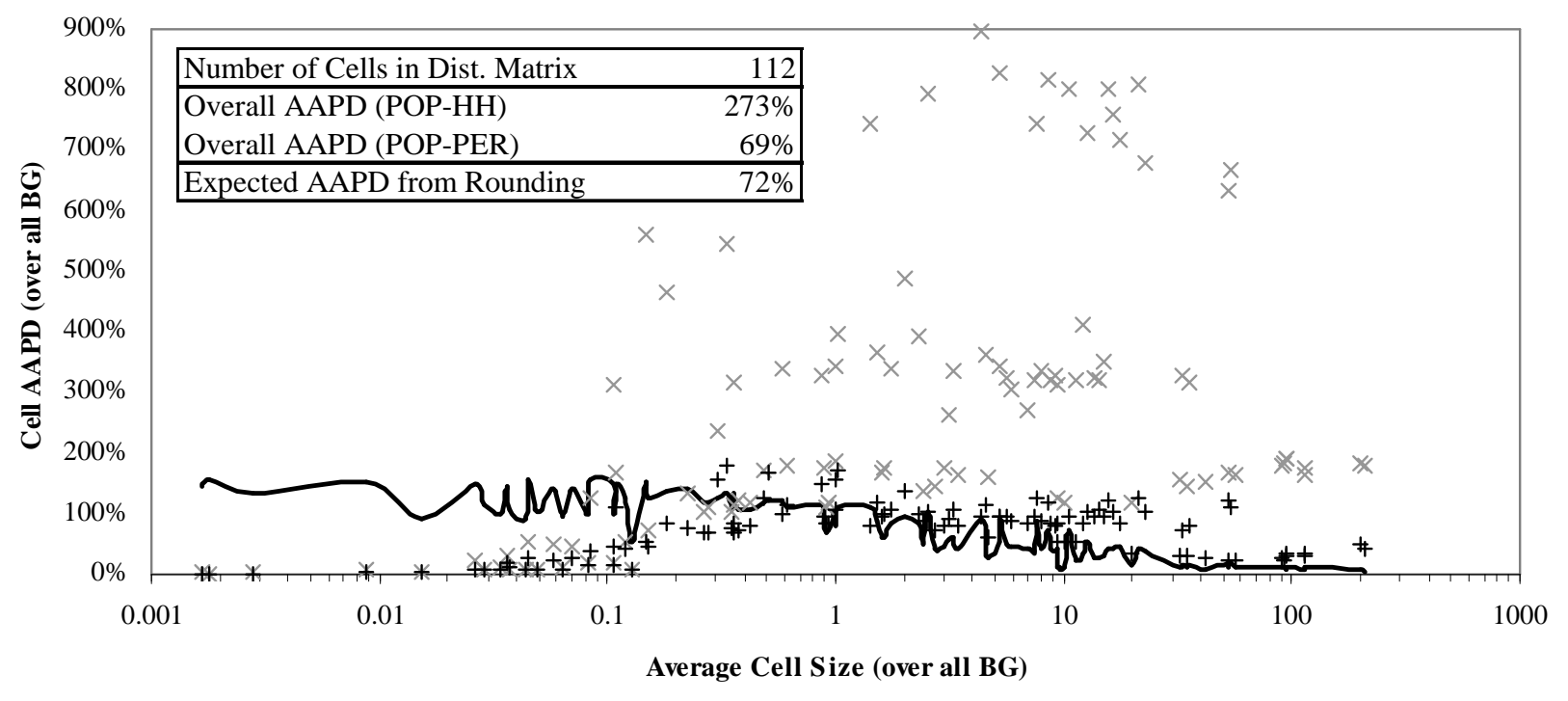

$\times$ AAPD $($ POP-HH) + AAPD (POP-PER) $\longrightarrow$ Expected AAPD

(b)

FIGURE 2 AAPD Comparison for (a) Household and (b) Person-Level Joint Distribution 